2007

\title{
Self-Consistent Analyses For Potential Conduction Block in Nerves By An Ultrashort High-Intensity Electric Pulse
}

\author{
R. P. Joshi \\ Old Dominion University \\ A. Mishra \\ Old Dominion University \\ Q. $\mathrm{Hu}$ \\ Old Dominion University \\ K. H. Schoenbach \\ Old Dominion University
}

A. Pakhomov

Old Dominion University, apakhomo@odu.edu

Follow this and additional works at: https://digitalcommons.odu.edu/bioelectrics_pubs

Part of the Biomedical Commons, Cell Biology Commons, and the Neurology Commons

\section{Repository Citation}

Joshi, R. P.; Mishra, A.; Hu, Q.; Schoenbach, K. H.; and Pakhomov, A., "Self-Consistent Analyses For Potential Conduction Block in Nerves By An Ultrashort High-Intensity Electric Pulse" (2007). Bioelectrics Publications. 232.

https://digitalcommons.odu.edu/bioelectrics_pubs/232

\section{Original Publication Citation}

Joshi, R. P., Mishra, A., Hu, Q. Schoenbach, K. H., \& Pakhomov, A. (2007). Self-consistent analyses for potential conduction block in nerves by an ultrashort high-intensity electric pulse. Physical Review E, 75(6), 061906. doi:10.1103/PhysRevE.75.061906

This Article is brought to you for free and open access by the Frank Reidy Research Center for Bioelectrics at ODU Digital Commons. It has been accepted for inclusion in Bioelectrics Publications by an authorized administrator of ODU Digital Commons. For more information, please contact digitalcommons@odu.edu. 


\title{
Self-consistent analyses for potential conduction block in nerves by an ultrashort high-intensity electric pulse
}

\author{
R. P. Joshi, ${ }^{1,2}$ A. Mishra, ${ }^{1}$ Q. Hu, ${ }^{1}$ K. H. Schoenbach, ${ }^{1,2}$ and A. Pakhomov ${ }^{3}$ \\ ${ }^{1}$ Department of Electrical \& Computer Engineering, Old Dominion University, Norfolk, Virginia 23529-0246, USA \\ ${ }^{2}$ Frank Reidy Center for Bio-Electrics, 830 Southampton Avenue, Norfolk, Virginia 23510, USA \\ ${ }^{3}$ General Dynamics Advanced Information Systems, Brooks City-Base, San Antonio, Texas 78235, USA
}

(Received 18 September 2006; published 7 June 2007)

\begin{abstract}
Simulation studies are presented that probe the possibility of using high-field $(>100 \mathrm{kV} / \mathrm{cm})$, short-duration $(\sim 50 \mathrm{~ns})$ electrical pulses for nonthermal and reversible cessation of biological electrical signaling pathways. This would have obvious applications in neurophysiology, clinical research, neuromuscular stimulation therapies, and even nonlethal bioweapons development. The concept is based on the creation of a sufficiently high density of pores on the nerve membrane by an electric pulse. This modulates membrane conductance and presents an effective "electrical short" to an incident voltage wave traveling across a nerve. Net blocking of action potential propagation can then result. A continuum approach based on the Smoluchowski equation is used to treat electroporation. This is self-consistently coupled with a distributed circuit representation of the nerve dynamics. Our results indicate that poration at a single neural segment would be sufficient to produce an observable, yet reversible, effect.
\end{abstract}

DOI: 10.1103/PhysRevE.75.061906

PACS number(s): 87.15.Aa, 87.50.Rr, 87.17.Aa

\section{INTRODUCTION}

It is well known that the application of electrical voltages (both dc and high-frequency) or, equivalently, the use of external current injection and extraction can reversibly block the conduction of action potentials across nerves [1-7]. Though other methods of achieving nerve conduction blockage such as pressure application [8], temperature lowering [9], and chemical and pharmacological means [5] exist, none can be as quick acting, localized, and yet reversible as electrical stimulation. Cessation of biological electrical signaling pathways can have a variety of applications in neurophysiology, clinical research, neuromuscular stimulation therapies, and even nonlethal bioweapons development. For example, pudendal nerve conduction block during micturition can reduce urethral pressure $[10,11]$ or help relieve chronic pain from a site of peripheral nerve injury [12]. It is well known that the nerve blocking ability of electrical stimulation is progressive from larger to smaller fibers $[13,14]$ and has been used to activate muscles in a physiological recruitment order and to reduce muscle fatigue. The concept of arresting action potential (AP) propagation on command through external electrical stimulation could open up the possibility of temporary incapacitation with applications to crowd control.

The generation and propagation of an action potential in a nerve fiber is triggered by perturbations in trans-membrane potential that activate ionic flows through voltage-gated sodium and potassium channels. Dynamical details and mathematical analyses of the various ionic currents across the cell membrane can be obtained from the Hodgkin-Huxley [15] and Huxley-Frankenhaeuser [16] models for unmyelinated and myelinated nerves, respectively. Action potential generation and propagation requires the attainment of a transmembrane voltage shift that exceeds a threshold level [17-20].

Since a threshold shift is a necessary requirement to initiate and maintain electrical propagation through a nerve fi- ber, any event that disrupts the trans-membrane voltage can potentially impede action potential propagation. One possibility is through the application of an external dc bias near a nerve. For a propagating action potential (initiated, for example, by a depolarizing voltage), the application of a positive bias on the outer region of the nerve would prevent the local potential from reaching the requisite negative value. This would effectively arrest AP propagation and hence, in theory, block nerve conduction. However, a number of potential and practical problems arise from the application of an external dc bias for purposes of a conduction block. (i) First, prolonged application of the dc bias can itself inject localized currents and charge the axonal membranes, thereby launching its own AP. The duration and amplitude of the external dc have to be sufficiently low to circumvent such "self-launch" phenomena. (ii) In addition, since the timing and sequence of propagating action potentials are not known a priori, it is practically very difficult to achieve reliable conduction blockages for all possible propagating APs. (iii) Any sharp rise times for the dc biasing voltages can lead to large capacitive charging currents that have a similar undesirable effect of self-launching an AP. Hence, the rise and fall times of any applied dc bias need to be sufficiently large. (iv) Long durations or repetitive dc biasing can potentially cause tissue damage due to internal heating [21]. For effective suppression of this deleterious effect, the net energy needs to be sufficiently small.

The application of high-frequency blocking signals alleviates some of the problems with dc biasing. Heat generation can be reduced and the biphasic signals make it somewhat more difficult to self-launch action potentials. However, the overall difficulties are not eliminated and the fundamental issues remain. In addition, the frequency of operation begins to play an important role in the blocking effectiveness. A frequency bandwidth limitation for AP extinction exists, and excitation that is either too fast or too slow cannot provide a conduction block [3]. This is easily understood from the 
standpoint of having to cause sufficient disruption of the trans-membrane potential during the time an incident AP approaches the "blocking spot." A very-low-frequency bias signal will not have sufficient time to disrupt an approaching action potential, while a very fast oscillatory signal will effectively present a net zero average perturbation. Furthermore, the continuous ac signal contributes to localized heating. For these above reasons, the use of ac biasing techniques for AP suppression is not really an optimal solution. Though various different wave forms and electrode arrangements have been proposed $[22,23]$, the underlying problems remain.

Almost all modeling and experimental work relating to AP conduction blocking (whether dc or high-frequency) has been performed at relatively low voltage amplitudes with temporal durations in the microsecond range or higher. However, the use of electric pulses with very high fields $(\sim 100 \mathrm{kV} / \mathrm{cm}$ or higher) and pulse durations in the nanosecond range [24-26] has been a very recent development in bioelectrics. From a practical standpoint, such high-intensity, short-duration electrical pulses (HISDEPs) have been shown to be useful for various biological applications ranging from cellular electroporation [27], electrically triggered intracellular calcium release $[28,29]$, the destruction of microorganisms [27,30,31], killing of tumor cells [27,32], DNA damage [33], and possibly wound healing [34,35]. The hallmark of such a HISDEP is the creation of a high density of nanometer-sized pores on the cellular membrane, followed by their recovery through resealing. This has been confirmed by analytical calculations [36-39] as well as molecular dynamic simulations [40-42] and verified by tracking fluorescent dyes in flow cytometry experiments [27]. Typical pore diameters are on the order of $1.6 \mathrm{~nm}$ with about a $0.6-\mathrm{nm}$ statistical spread. Time scales for pore resealing range from milliseconds to a few seconds [36,43-47] with the dynamical details depending on parameters such as cell diameter, applied electric field magnitude, permittivities, and membrane tension.

Here we discuss and analyze the possibility of applying such HISDEPs for blocking nerve conduction by modulating the membrane conductivity through the electroporation process. Such a study has not been presented to the best of our knowledge, though simple considerations of electroporation in the context of ventricular muscle breakdown were reported [48]. Formation of a high pore density would increase the local membrane conductivity and effectively "short-out" the trans-membrane potential of a nerve in the vicinity of the pulsing electrode. The net effect would be a disruption in the requisite trans-membrane potential shift required to sustain AP propagation. The use of an HISDEP in conduction block context would conceivably offer the following advantages: (a) negligible heating, due to the low energy content of each pulse. This would minimize any possible tissue damage and allow repetitive pulsing (even multiple firings with tailored amplitudes) to achieve the desired effects for selected durations. (b) The effects of such HISDEPs would be reversible due to the resealing of pores created electrically by the external voltage. Hence, a permanent effect would be avoided. (c) The turn-on would be relatively fast (on the order of tens of nanoseconds [36]), and the effect could be spatially tai- lored. (d) Unlike the dc or ac excitation, it would be more difficult to "self-launch" action potential waves, which required depolarization over microsecond time scales or longer. The porated regions of the membrane would effectively clamp the local trans-membrane potential to near-zero values. Also, the nanosecond pulse termination would produce a negative voltage change with time and, hence, a strong negative displacement current. This would cause the membrane potentials to fall sharply back to their resting potentials well before the microsecond time scales that are typically required for launching action potentials.

The mathematical details and quantitative analyses of such AP blockage in response to a HISDEP are modeled and simulated. The primary thrust of our contribution is on an unexplored aspect of nanosecond, high-intensity electric pulses with potential applications to disruption of neural traffic. Theoretical analyses and predictions are presented here as a first step. We hope to discuss detailed experimental data elsewhere from studies already underway within our group. Experimental details and many of the practical issues have thus not been discussed here. For self-consistency, pore formation dynamics and the resulting evolution in membrane conductivities fashioned by a nanosecond-duration, highvoltage pulse are first calculated. These are then embedded into a McNeal-type cable model [49] for electrical conduction along a nerve. A cylindrical geometry with a constant cross section has been assumed for simplicity without compromising the qualitative physics. An additional shunt membrane conductance in parallel to the ionic channel currents is included to account for the time-dependent flow through the electropores.

\section{SIMULATION DETAILS}

\section{A. Cable model of nerve propagation}

The theoretical basis for modeling electrostimulation of excitable nerve tissues is briefly discussed to highlight the changes necessary for incorporating the effects of electroporation. Electrical propagation in nerves was first discussed by Cooley and Dodge [50] and later by McNeal [49] and Rattay [51] based on a distributed, transmission-line model. In a one-dimensional treatment, the entire nerve is discretized spatially into nodes as shown in Fig. 1 and the transmembrane potential at any time for a given node $n$ expressed as

$$
\begin{aligned}
& \quad\left[V_{i-1}(t)-V_{i}(t)\right] / R_{a}-\left[V_{i}(t)-V_{i+1}(t)\right] / R_{a}+I_{\text {inj }}(t)-I_{\mathrm{ch}}(t) \\
& \quad=d\left[C_{m}(t) V_{i}(t)\right] / d t,
\end{aligned}
$$

where $V_{i}(t)$ is the net trans-membrane potential at node $i, R_{a}$ the effective axonal resistance, and $C(t)$ the effective timedependent membrane capacitance that incorporates dynamic pore formation. $I_{\mathrm{inj}}(t)$ and $I_{\mathrm{ch}}(t)$ denote the injected stimulus current that initiates a propagating action potential and membrane channel current, respectively, at node $i$ during time instant $t$. As shown in Fig. 1, the channel current at any node $i$ consists of the usual sodium and potassium ionic currents through voltage-gated channels and a leakage contribution given mathematically as $[15,16]$ 


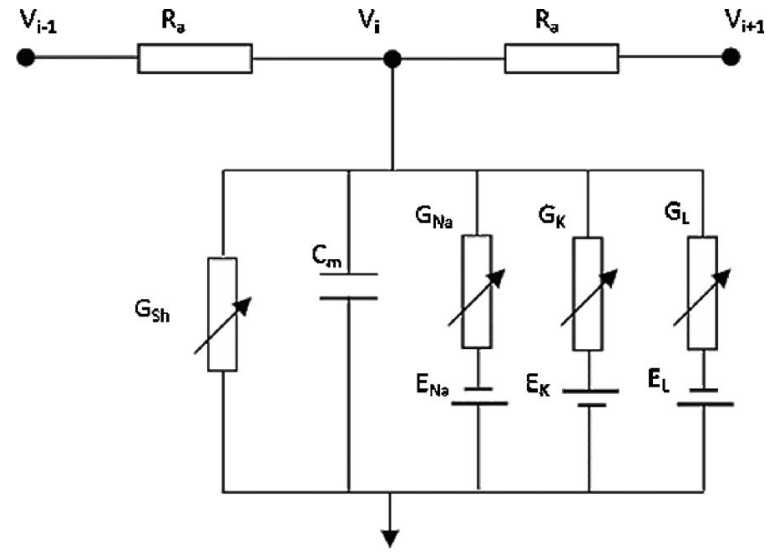

FIG. 1. Schematic of the cable model used to represent a nerve fiber.

$$
\begin{aligned}
I_{\mathrm{ch}}(t)= & g_{\mathrm{Na}} m_{i}^{3}(t) h_{i}(t)\left[V_{i}(t)-V_{\mathrm{Na}}(t)\right]+g_{\mathrm{K}} n_{1}^{4}(t)\left[V_{i}(t)-V_{\mathrm{K}}(t)\right] \\
& +g_{L}\left[V_{i}(t)-V_{L}(t)\right]+G_{\mathrm{sh}}(t) V_{i}(t),
\end{aligned}
$$

where $g_{\mathrm{Na}}$ and $g_{\mathrm{K}}$ are the sodium- and potassium-channel conductances, while $V_{\mathrm{Na}}(t)$ and $V_{\mathrm{K}}(t)$ denote the resting potentials. In order to account for the effects of electroporation, an additional time-dependent shunt conductance $G_{\mathrm{sh}}(t)$ has been included in Eq. (2) and placed as a parallel element in the circuit of Fig. 1. The terms $m_{i}(t)$ and $h_{i}(t)$ represent the time-varying switching variables for the activation and deactivation of sodium channels across the membrane at the $i$ th discrete location, while $n_{i}(t)$ denotes the potassium channel activating switch.

Strictly, ionic transfer across the membrane is a stochastic process [52], and individual channels randomly transition between open and closed states. These rates of transition are voltage dependent, leading to a net change in ionic transport upon electrical stimulation. The collective macroscopic behavior, however, can be expressed in terms of a continuum time-dependent model for all channels. Such a global approach, first suggested by Hodgkin-Huxley [15] for unmyelinated nerves, has been used here and leads to the following dynamical equations for the switching functions $m(t), h(t)$, and $n(t)$ :

$$
\begin{gathered}
d\left[m_{i}(t)\right] / d t=\alpha_{m}(t)\left\{1-m_{i}(t)\right\}-\beta_{m}(t) m_{i}(t), \\
d\left[n_{i}(t)\right] / d t=\alpha_{n}(t)\left\{1-n_{i}(t)\right\}-\beta_{n}(t) n_{i}(t), \\
d\left[h_{i}(t)\right] / d t=\alpha_{h}(t)\left\{1-h_{i}(t)\right\}-\beta_{h}(t) h_{i}(t) .
\end{gathered}
$$

In Eq. (3) above, the voltage-dependent transition rates $\alpha_{m, n, h}$ and $\beta_{m, n, h}$ are given as

$$
\alpha_{m}(t)=\phi(T)\left[2.5-0.1 V_{i}(t)\right] /\left\{\exp \left[2.5-0.1 V_{i}(t)\right]-1\right\},
$$

$$
\alpha_{n}(t)=\phi(T) 0.1\left[1-0.1 V_{i}(t)\right] /\left\{\exp \left[1-0.1 V_{i}(t)\right]-1\right\},
$$

$$
\alpha_{h}(t)=\phi(T) 0.07\left\{\exp \left[-V_{i}(t) / 20\right]\right\},
$$

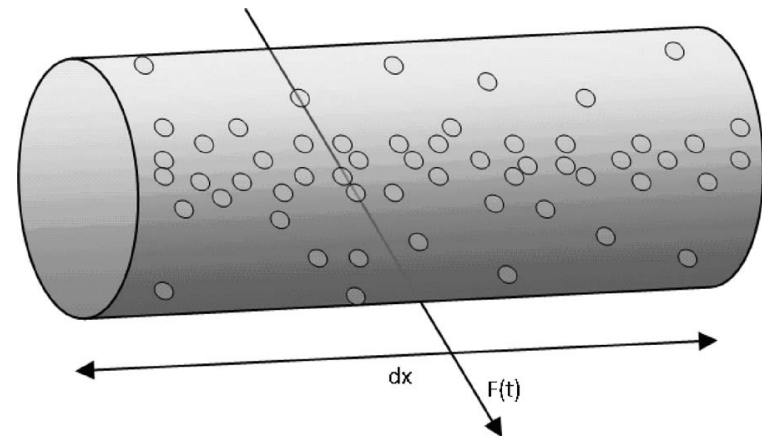

FIG. 2. Sketch of pores created on the nerve membrane by the external voltage. A differential $d x$ of the nerve fiber is shown, and the external field is normal to the longitudinal axis.

$$
\begin{gathered}
\beta_{m}(t)=\phi(T) 4\left\{\exp \left[-V_{i}(t) / 18\right]\right\}, \\
\beta_{n}(t)=\phi(T) 0.125\left\{\exp \left[-V_{i}(t) / 80\right]\right\}, \\
\beta_{h}(t)=\phi(T) /\left\{\exp \left[3-0.1 V_{i}(t)\right]+1\right\},
\end{gathered}
$$

and

$$
\phi(T)=3^{(T-6.3) / 10}
$$

where $T$ is the temperature in degrees centigrade. Due to external voltage pulsing, each node voltage $V_{i}(t)$ is time dependent, and hence, the transition rates also become dynamic. For numerical stability and accuracy, the implicit centered-difference scheme [50] can be used for updating the functions $m_{i}(t), n_{i}(t)$, and $h_{i}(t)$ at each succeeding time step. This, for example, leads to the following discretized equation for $m_{i}(t+\Delta t)$ after a time step $\Delta t$ :

$$
\begin{aligned}
m_{i}(t+\Delta t)= & m_{i}(t)+(\Delta t / 2)\left\{\alpha_{m, i}(t)\left[1-m_{i}(t)\right]+\alpha_{m, i}(t+\Delta t)\right. \\
& \left.-\beta_{m, i}(t) m_{, i}(t)\right\} /\left\{1+(\Delta t / 2)\left[\alpha_{m, i}(t+\Delta t)\right.\right. \\
& \left.\left.+\beta_{m, i}(t+\Delta t)\right]\right\} .
\end{aligned}
$$

\section{B. Membrane electroporation effects}

The above set of equations (1)-(5) yields time-dependent voltages at each nerve node and constitutes the usual approach for analyses of propagating action potentials. The application of a HISDEP leads to nanopore formation on the cylindrical membrane surface as sketched in Fig. 2. As a result, the traditional cable model needs to be modified to take account of the increased membrane conductance [the $G_{\text {sh }}(t)$ term of Eq. (2)] and the altered membrane capacitance [the $C_{m}(t)$ term of Eq. (1)]. These parameters characterizing the electrical response are, in general, dictated by the number of pores (conduction pathways) formed for a given voltage amplitude and pulse duration. Hence, the foremost task is the evaluation of the time- and voltage-dependent pore density. The dynamics of pore creation and destruction have been well characterized by continuum approaches based on the Smoluchowski equation [36-39,53-62], though rigorously microscopic molecular-dynamics (MD) treatments are more accurate. However, given the computational complexity and 
large memory requirements of the MD technique, a meanfield continuum numerical model will be adopted here.

The mathematical equation governing the pore dynamics based on the continuum Smoluchowski theory is given in terms of the pore density distribution function $n(r, t)$ as

$$
\begin{gathered}
\subset n(r, t) / \subset t-\left[D /\left(k_{B} T\right)\right]\{\subset[n(r, t) \subset E(r) / \subset r] / \subset r\} \\
-D\left[\subset^{2} n(r, t) / \subset r^{2}\right]=S(r),
\end{gathered}
$$

where $S(r)$ is the source (or pore formation) term, $D$ is a pore diffusion constant, $T$ is temperature in Kelvin, and $k_{B}$ the Boltzmann constant. The process of diffusion represents a "random walk" of the pore radius in " $r$ space." Physically, this is brought about by fluctuations in the radius in response to water molecules and other species constantly entering and leaving the pores. Two types of pores (hydrophilic and hydrophobic) are known to exist $[58,63,64]$. The formation of pores is generally assumed to be a two-step process. All pores are initially created as hydrophobic and nonconducting at a rate $S(r)$ per unit area of the membrane, during every time interval $d t$. This rate is given by

$$
S(r)=\left[\left(v_{c} h\right) /\left(k_{B} T\right)\right][d E(r) / d r] \exp \left[-E(r) /\left(k_{B} T\right)\right] d r,
$$

where $v_{c}$ is an attempt rate density [52], $E(r)$ the energy for hydrophobic pores, $T$ the operating temperature, and $k_{B}$ the Boltzmann constant. This assumes that the use of a kinetic collisional theory remains valid for nongaseous phases as well. If a nonconducting pore is created with a radius $r>r^{*}$ $(=0.5 \mathrm{~nm})$, it spontaneously changes its configuration and transforms into a conducting, hydrophilic pore. All conducting pores then survive as long as their radii remains larger than $r^{*}$. Destruction of a conducting pore occurs only if it drifts or diffuses in $r$ space to a value below $r^{*}$. Due to the exponential term in Eq. (7), most pores are created with very small radii.

Each of the two pore types is characterized by an energy of formation, $E(r)$, which is a function of the pore radius $r$. The energy function $E(r)$ determines the "drift flux" for pores in $r$ space and, therefore, governs the growth or contraction of pores at any given radius $r$. This function $E(r)$ depends on several factors, including the membrane tension, the applied voltage and associated stored electrostatic energy, and steric repulsion. The published and accepted model for $E(r)$ provides the following pore energy function $[53,63,65,66]$ :

$$
E(r)=2 \pi h r \sigma(\infty)\left[I_{1}\left(r / r_{0}\right) / I_{0}\left(r / r_{0}\right)\right]-\pi \alpha_{p} V^{2} r^{2}
$$

and

$$
E(r)=2 \pi \gamma r-\left(\int_{0}^{r} 2 \pi \Gamma\left(r^{*}\right) r^{*} d r^{*}\right)+(C / r)^{4}-\pi \alpha_{p} V^{2} r^{2},
$$

for hydrophobic and hydrophilic pores, respectively. In the above, $I_{1}$ and $I_{0}$ are the modified Bessel functions of the zeroth and first order, respectively, $h$ is the membrane thickness, $\sigma(\infty)$ is a constant on the order of $5 \times 10^{-2} \mathrm{~N} \mathrm{~m}^{-1}$ [65], while $r_{0}$ represents a characteristic length scale over which the properties of water change between the interface and the bulk. The value of $r_{0}$ is taken to equal $1 \mathrm{~nm}$ [58]. The $(C / r)^{4}$ term in Eq. (8b) accounts for steric repulsion between the lipid heads lining the pore and contributes to an increase in energy with shrinking radius [58,67]. A typical value for $C$ has been reported to be about $9.67 \times 10^{-15} \mathrm{~J}^{0.25} \mathrm{~m}$ [58]. The last term in Eq. (8b) represents the capacitive contribution to the energy in the presence of a trans-membrane potential $V$. The coefficient $a_{p}$ is a property of the membrane and its aqueous environment. In the simplest continuum approximation [65], it is expressed in terms of the membrane thickness $h$ and the permittivities $\varepsilon_{w}$ and $\varepsilon_{m}$ of water and the membrane, respectively, as $a_{p}=\left(\varepsilon_{w}-\varepsilon_{m}\right) /[2 h]$. It might be mentioned that other models that take into account pore conductivity and ionic distortions of the electric field $[54,59]$ have been proposed for the electrostatic energy calculations. Finally, $\gamma$ is the energy per unit length of the pore perimeter, while $\Gamma$ is the energy per unit area of the intact membrane. Most analyses in the literature [58] use a constant surface tension parameter $\left(\Gamma=\Gamma_{0}\right)$, yielding the following simplified formation energy expression for conducting pores:

$$
E(r)=2 \pi \gamma r-\pi \Gamma_{0} r^{2}+(C / r)^{4}-\left[\left(\varepsilon_{w}-\varepsilon_{m}\right) / 2 h\right] \pi r^{2} V^{2},
$$

though more complex dynamic extensions have been reported [39].

The above set of equations provides for an evaluation of the pore probability density distribution $n(r, t)$ at each time step, in response to a given time-dependent trans-membrane potential $V(t)$. The total number of pores, $N(t)$, can then be obtained from the density function $n(r, t)$ as $N(t)=\int_{0}^{\infty}$ $n(r, t) d r$. Similarly, the average pore radius $\langle r(t)\rangle$ at any time instant is then $\langle r(t)\rangle=\left[\int_{0}^{\infty} r n(r, t) d r\right] /\left[\int_{0}^{\infty} n(r, t) d r\right]$. Pore creation also changes the membrane capacitance since water is able to enter the aqueous pathways in the membrane and alter the local permittivity. In this context, the effective capacitance $C_{m}(t)$ per unit longitudinal distance $d z$ is given as

$$
\begin{aligned}
C_{m}(t)= & \frac{\varepsilon_{w} A_{p}+\varepsilon_{m} A_{\mathrm{mem}}}{h} \\
= & \frac{\varepsilon_{w}}{h}\left(d z \int_{0}^{\pi}\left(R_{i}+R_{o}\right) d \theta \int_{0}^{\infty} n(r, \theta, t) d r\right) \\
& +\frac{\varepsilon_{m}}{h}\left(\pi\left(R_{i}+R_{o}\right) d z\right. \\
& \left.-d z \int_{0}^{\pi}\left(R_{i}+R_{o}\right) d \theta \int_{0}^{\infty} n(r, \theta, t) d r\right),
\end{aligned}
$$

where $A_{p}$ and $A_{\text {mem }}$ are the pore area and membrane surface area, respectively, while $R_{i}$ and $R_{o}$ are the inner and outer radii of the nerve fiber (membrane thickness $h=R_{o}-R_{i}$ ).

\section{Membrane potential calculations for electroporation}

The problem of determining the number of pores on the nerve membrane that facilitate conduction and, hence, control the $G_{\text {sh }}$ of Eq. (2) can be completely resolved provided 


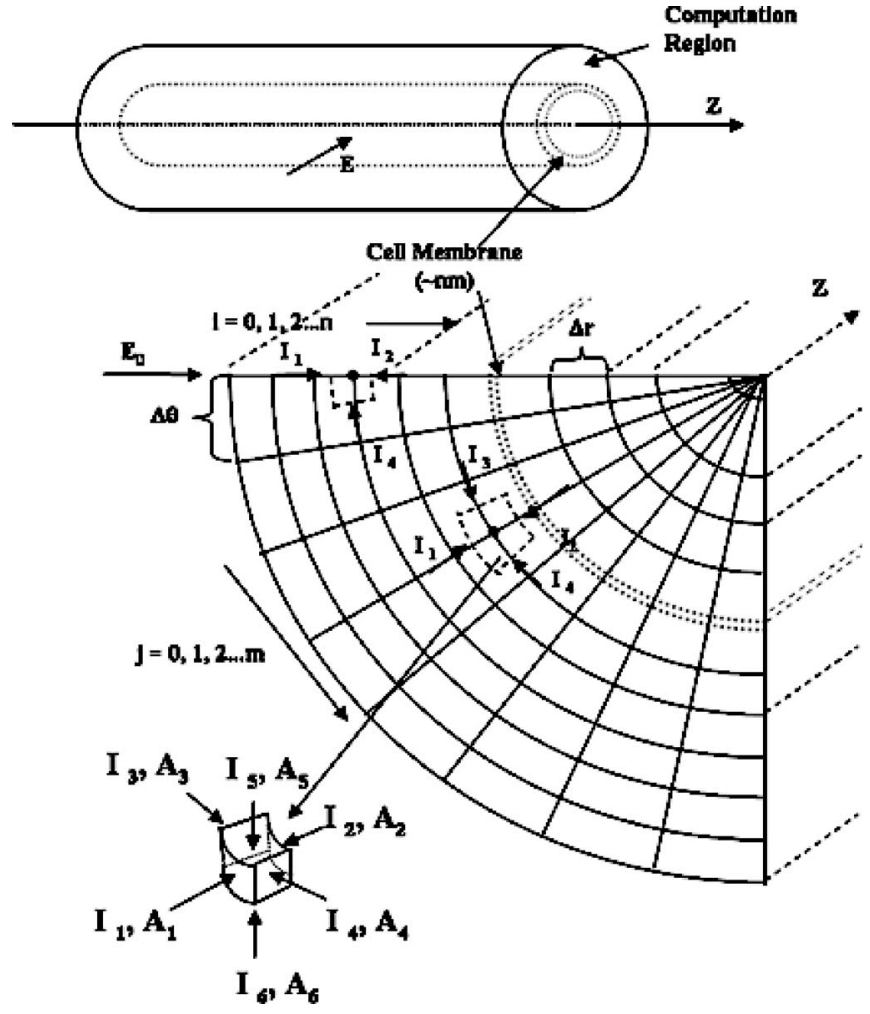

FIG. 3. Schematic of one-quarter of the model used to represent a cable model of a nerve cell for the distributed electrical calculations. The dotted box shows a typical element with current flows.

the time-dependent trans-membrane potential that drives pore creation is known. Towards this end, the following approach based on a time-dependent solution was adopted for calculations of the trans-membrane potential. The external electric field $F(t)$ was taken to be time dependent, allowing for the exact modeling of actual external pulsed wave forms.

An approach for calculating the trans-membrane potential is through a time-domain nodal analysis involving a distributed equivalent circuit representation of a cell and its membrane structures. The dynamic membrane electric field can then be obtained from the time-dependent trans-membrane voltage. Details of this method applied to spherical cell and its implementation have been given elsewhere [38,41], and hence, only a brief outline will be discussed here. Essentially, the entire cell volume was broken up into finite segments, and each segment represented by a parallel $R C$ combination to account for the current flow and charging effects. The computational region was a cylinder that included the cell, its sub structure, and surrounding suspension medium, discretized along the $r$ and $\theta$ directions as shown in the cross section of Fig. 3. Longitudinal (z-directional) symmetry was used to discard the $z$ coordinate, while retaining only the radial $(r)$ and angular $(\theta)$ coordinates of a cylindrical system. For simplicity, the plasma membrane was taken as an integral unit; i.e., this subregion was not further discretized. Boundary nodes are treated separately. For interior nodes, the current continuity equation is of the form

$$
\sum_{k=1}^{6}\left(\sigma E+\varepsilon \frac{\partial E}{\partial t}\right)_{k} A_{k}=\sum_{k=1}^{6} I_{k}=0,
$$

where $I_{5}$ and $I_{6}$ are currents along the $z$ direction, $A_{k}$ the surface area, $E$ the electric field, $\varepsilon$ the permittivity, and $\sigma$ the conductivity. Considerations of geometric symmetry of the computation region lead to $I_{5}=I_{6}=0$ due to the equipotentials. In order to reduce the computation load, only a quarter of the entire spherical computational region was considered based on the inherent symmetry. Nodes with $j=0$ and $j=m$ had to be treated carefully. Only $I_{1}, I_{2}$, and $I_{4}$ are nonzero since the targeted element only has five faces as face 3 shrinks to a line. For $j=0$ and $0<i<n$, Eq. (11) effectively becomes

$$
\begin{gathered}
\sigma_{1} \frac{V_{i-1, j}^{t}-V_{i, j}^{i}}{\Delta r} A_{1}+\sigma_{2} \frac{V_{i+1, j}^{i}-V_{i, j}^{t}}{\Delta r} A_{2}+\sigma_{3} \frac{V_{i, j-1}^{t}-V_{i, j}^{t}}{r \Delta \theta} A_{3} \\
+\sigma_{4} \frac{V_{i, j+1}^{t}-V_{i, j}^{t}}{r \Delta \theta} A_{4}+\frac{\varepsilon_{1}}{\Delta t}\left(\frac{V_{i-1, j}^{t+1}-V_{i, j}^{t+1}}{\Delta r}-\frac{V_{i, 1, j}^{t}-V_{i, j}^{t}}{\Delta r}\right) A_{1} \\
+\frac{\varepsilon_{2}}{\Delta t}\left(\frac{V_{i+1, j}^{t+1}-V_{i, j}^{t+1}}{\Delta r}-\frac{V_{i+1, j}^{t}-V_{i, j}^{t}}{\Delta r}\right) A_{2}+\frac{\varepsilon_{3}}{\Delta t}\left(\frac{V_{i, j-1}^{t+1}-V_{i, j}^{t+1}}{r \Delta \theta}\right. \\
\left.\quad-\frac{V_{i, j-1}^{t}-V_{i, j}^{t}}{r \Delta \theta}\right) A_{3}+\frac{\varepsilon_{4}}{\Delta t}\left(\frac{V_{i, j+1}^{t+1}-V_{i, j}^{t+1}}{r \Delta \theta}-\frac{V_{i, j+1}^{t}-V_{i, j}^{t}}{r \Delta \theta}\right) A_{4} \\
=0 .
\end{gathered}
$$

Here $A_{1}=(r+\Delta r / 2) \Delta \theta \Delta z, A_{2}=(r-\Delta r / 2) \Delta \theta \Delta z$, and $A_{3}=A_{4}$ $=\Delta r \Delta z$. Putting $A_{1}-A_{4}$ into Eq. (2), we get

$$
\begin{aligned}
\sigma_{1} \frac{V_{i-1, j}^{t}-V_{i, j}^{t}}{\Delta r}(r+\Delta r / 2) \Delta \theta+\sigma_{2} \frac{V_{i+1, j}^{t}-V_{i, j}^{t}}{\Delta r}(r-\Delta r / 2) \Delta \theta \\
+\sigma_{3} \frac{V_{i, j-1}^{t}-V_{i, j}^{t} \Delta r+\sigma_{4} \frac{V_{i, j+1}^{t}-V_{i, j}^{t}}{r \Delta \theta} \Delta r}{r \Delta \theta} \\
+\frac{\varepsilon_{1}}{\Delta t}\left(\frac{V_{i-1, j}^{t+1}-V_{i, j}^{t+1}}{\Delta r}-\frac{V_{i-1, j}^{t}-V_{i, j}^{t}}{\Delta r}\right)(r+\Delta r / 2) \Delta \theta \\
+\frac{\varepsilon_{2}}{\Delta t}\left(\frac{V_{i+1, j}^{i+1}-V_{i, j}^{t+1}}{\Delta r}-\frac{V_{i, 1, j}^{t}-V_{i, j}^{t}}{\Delta r}\right)(r-\Delta r / 2) \Delta \theta \\
+\frac{\varepsilon_{3}}{\Delta t}\left(\frac{V_{i, j-1}^{t+1}-V_{i, j}^{t+1}}{r \Delta \theta}-\frac{V_{i, j-1}^{t}-V_{i, j}^{t}}{r \Delta \theta}\right) \Delta r \\
+\frac{\varepsilon_{4}}{\Delta t}\left(\frac{V_{i, j+1}^{t+1}-V_{i, j}^{t+1}}{r \Delta \theta}-\frac{V_{i, j+1}^{t}-V_{i, j}^{t}}{r \Delta \theta}\right) \Delta r=0 .
\end{aligned}
$$

In the above, $V_{i, j}^{t}$ stands for the potential at node $(i, j)$ at time $t$ with $i=0,1 \ldots, n$ and $j=0,1, \ldots, m$. For an electric field applied along the $r$ direction, potential at node with $i=n$ is zero. Another boundary condition to be considered is for nodes with $i=0$. The potentials of such nodes are calculated as $V_{0, j}=-E_{0} R \cos \theta$, for $j=0, \ldots, m$, where $E_{0}$ is the externally applied electrical field and $R$ the radius of the computational region.

Combining with the boundary conditions discussed above, one gets $N$ equations for the $N$ unknown node voltages. These $N$ equations can easily be solved by any linear equation solver. Potentials on each node are easily updated at 
every time step based on the value of the externally applied field.

\section{Pore-facilitated membrane conductivity}

Membrane conductivity is modulated by pore formation and qualitatively depends on the number of pores, their effective radii, the energy barrier to flow produced by the ionic interactions between the pore and surrounding dielectric, and the spreading resistance. Treatments of pore conduction have been described in the literature $[36,65,68]$. While the treatment given below was originally discussed in the context of pulses longer than the nanosecond range, the basic physics of transport over a voltage-dependent barrier and pore conductivity holds true regardless. As long as the transmembrane potential is suitably calculated and applied, the expressions given next remain valid. Following Glaser et al. [65], the pore conductivity $G_{\text {pore }}$ was taken to be

$$
\begin{aligned}
G_{\text {pore }}(t)= & \pi R_{0}^{2} H N(t)\{\exp [U(t)]-1\} /\left(U(t) \int_{0}^{h} d x \exp [U(t)\right. \\
& \left.\left.\times(1-x / h)+W(x) / k_{B} T\right]\right),
\end{aligned}
$$

where $U(t)=\left\{V(t)^{*} q\right\} /\left(k_{B} T\right), q$ being the electronic charge, $H$ the conductivity of the aqueous solution, $V(t)$ the timedependent trans-membrane potential, $h$ the membrane thickness, $N(t)$ the number of pores, and $W(x)$ the energy barrier for an ion inside the pore. The $W(x)$ profile has a trapezoidal shape $[65,69]$ with a linearly varying region of thickness $d$ at the two ends of the pore and a constant value of $W_{0}$ over the central pore region. Thus, mathematically the profile of $W(x)$ is

$$
\begin{gathered}
W(x)=W_{0} \quad \text { for } d<x<(h-d), \\
W(x)=W_{0} x / d \quad \text { for } 0<x<d,
\end{gathered}
$$

and

$$
W(x)=W_{0}[(h-x) / d] \text { for }(h-d)<x<h .
$$

Typically $W_{0}$ is much larger than the thermal energy $k_{B} T$, and under these conditions, the pore conductivity simplifies to

$$
\begin{aligned}
G_{\text {pore }}(t)= & L_{z} \int_{0}^{\pi} d \theta \int_{0}^{\infty}\left[n(r, \theta, t) d r r^{2} H D / h\right] /\{[1+P(t)] \\
& \left.\times \exp \left[W_{0} /\left(k_{B} T\right)-\eta U(t)\right]-P(t)\right\},
\end{aligned}
$$

where $P(t)=[\eta U(t)] /\left[W_{0} /\left(k_{B} T\right)-\eta U(t)\right], \eta=d / h$ is a measure of the relative size of the pore, $D$ the diameter of the nerve fiber, and $L_{z}$ the internodal segment along the nerve. In Eq. (16), $n(r, \theta, t)$ is the pore density distribution function previously defined in Eq. (6). Since the number of pores is nonuniform with an angular dependence, the pore resistance needs to be evaluated across differential stripes of width $R d \theta$ ( $R=$ nerve fiber radius) and longitudinal length $L_{z}$ across the nerve membrane. The overall shunt conductivity $G_{\mathrm{sh}}$ is obtained by adding the spreading resistance at the pore entrance to the effective pore resistivity. Thus $G_{\mathrm{sh}}=\left[1 / G_{\mathrm{sh}}+R_{\mathrm{sp}}\right]^{-1}$

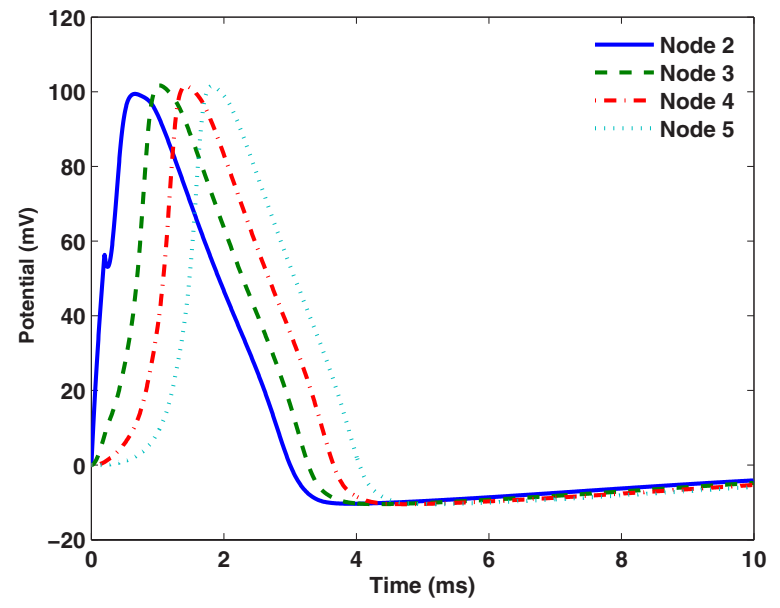

FIG. 4. (Color online) Numerical results showing propagating node potentials in a nonmyelinated nerve following excitation of node 2 .

where the pore spreading resistance $R_{\mathrm{sp}}=\rho / 2 r$ and is the resistivity of the aqueous medium. This latter is also referred to as the access resistance $[70,71]$.

\section{RESULTS AND DISCUSSION}

\section{A. Action potential propagation without electroporation}

Simulation results are first presented on AP propagation for an unmyelinated fiber in the absence of any nerve electroporation to provide a base line and allow for relative comparisons. Idealized conditions, such as uniform diameter nerves and simple geometric shapes, have been used in our simulations for simplicity. While the practical details are more complex, the general trends and qualitative physics discussed here should hold. Figure 4 shows the time-dependent potentials at various distinct nodes for a myelinated nerve. An axon diameter of $23.8 \mu \mathrm{m}$ was chosen with a specific resistance of $34.5 \Omega \mathrm{cm}$ and a specific membrane capacitance of $1 \mu \mathrm{F} \mathrm{cm}^{-2}$. The remaining parameters required in Eqs. (2)-(4) were assigned values usually reported in the literature $[3,49,50,72]$. The triggering excitation for launching the AP was through a $0.2-\mathrm{ms}-\mathrm{long}, 0.25-\mu \mathrm{A}$ pulse at node 2 of a 200-node section. As seen from Fig. 4, the maximum voltage at the various nodes is roughly fixed. The temporal shift allows for calculations of the propagation speed $v$. Results from several simulations carried out by varying the nerve diameter $d$ are listed in Table I. The best fit to the diameter dependent velocity was $v=6.4607 d^{0.517}$. This agrees well with the analytical predictions of a $v \sim d^{0.5}$ dependence [72]. Though not shown, the velocities in Table I were obtained for AP propagation from a single-shot triggering of the nerve. For multiple firings, the propagating velocity $v$ was slightly reduced after the first triggering due to the wellknown refractory period $[73,74]$. This occurs because a finite recovery time is required to regain initial equilibrium conditions (ion densities, potentials, etc.) within the nerves following an excitation.

Effects of applying an ac voltage for purposes of blocking a propagating AP are demonstrated next. A 9-kHz interrupt 
TABLE I. Computed conduction velocity for different axon diameters for unmyelinated fibers.

\begin{tabular}{lc}
\hline \hline Diameter $(\mathrm{cm})$ & Velocity $(\mathrm{cm} / \mathrm{ms})$ \\
\hline 0.0024 & 0.265957 \\
0.003 & 0.301205 \\
0.005 & 0.393701 \\
0.007 & 0.471698 \\
0.010 & 0.568182 \\
0.015 & 0.694444 \\
0.020 & 0.862069 \\
0.030 & 0.974659 \\
0.040 & 1.18765 \\
\hline \hline
\end{tabular}

signal of amplitude $4.0 \mu \mathrm{A}$ was applied continuously at node 17. As shown in Fig. 5(a), an AP is initiated at node 2 and propagates along both directions of the longitudinal nerve. The forward-traveling wave, upon reaching node 17 , is ef-
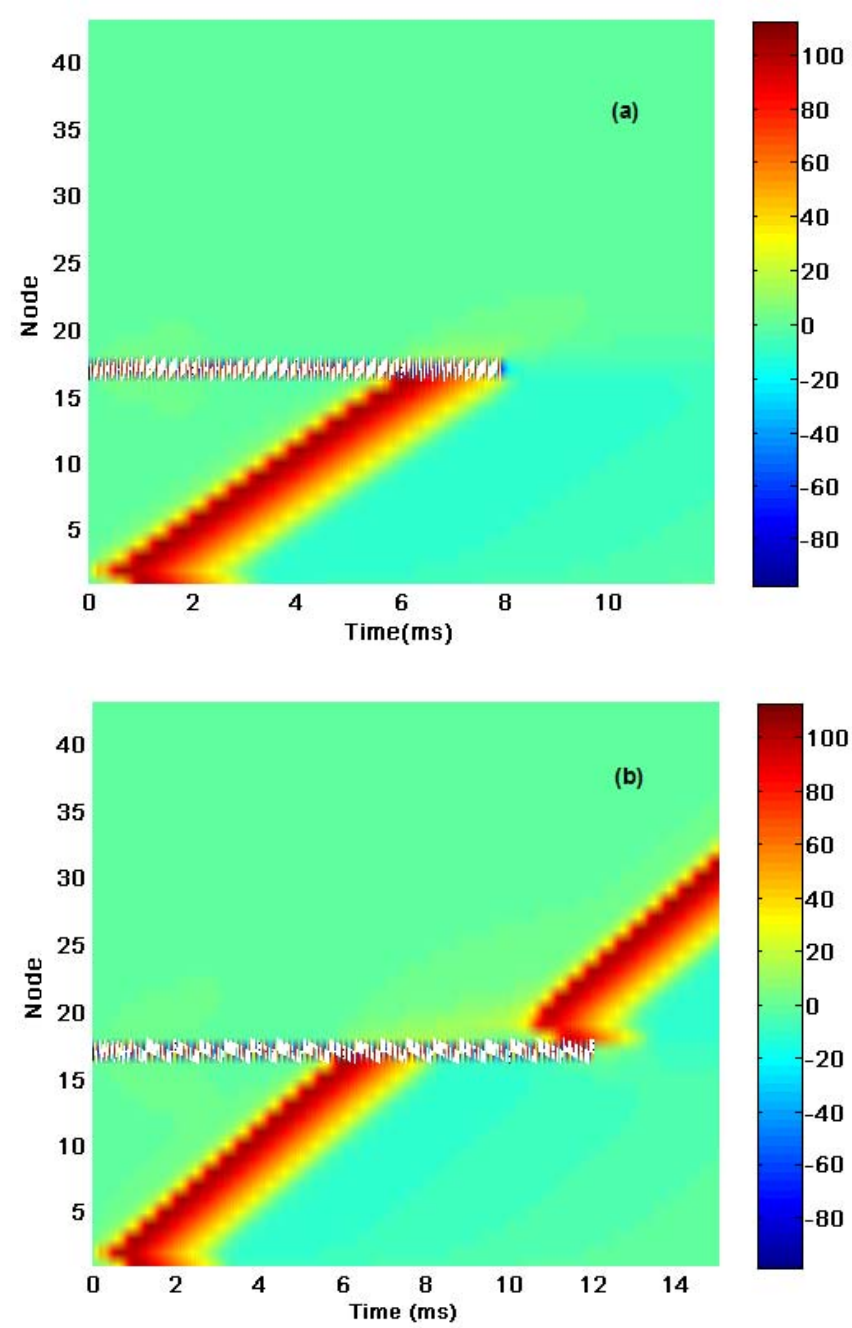

FIG. 5. (Color online) Simulations showing node voltage versus time with a 9-kHz ac block applied at node 17 and an AP launched from node 2. (a) ac signal terminated after $8 \mathrm{~ms}$ and (b) ac signal applied for a longer duration leading to self-launching of an AP.

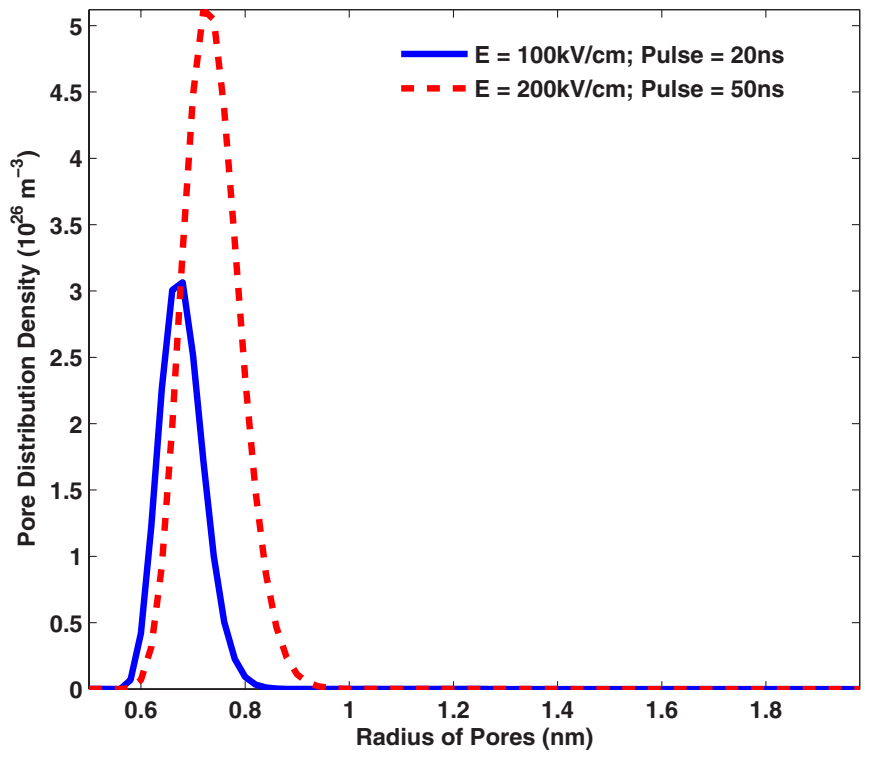

FIG. 6. (Color online) Simulations showing the distribution of pore radii at the end of two pulses of different field strengths and pulse widths.

fectively blocked. In order to prevent possible self-launch of any action potentials, the ac signal was terminated after $8 \mathrm{~ms}$ upon successful blockage. Figure 5(b) shows the result if the ac signal were not terminated, but instead continued until $12 \mathrm{~ms}$. Though an initial block at about $7 \mathrm{~ms}$ is achieved, a secondary AP is generated from node 17 due to the "selflaunch" mechanism of the ac signal itself after a finite delay. Thus, long-term application of an "ac block signal" is clearly inappropriate. These results are all in agreement with previous reports and display anticipated trends.

\section{B. Membrane electroporation effects}

Numerical evaluations of membrane pores and their dynamical evolution in response to an applied HISDEP were carried out for obtaining values of the shunt conductance $G_{\text {sh. }}$. As already discussed, the role of the HISDEP is to transform the neural fiber into a "locally shorted electrical element" that can no longer sustain the propagation of an electrical signal. This is analogous, in a loose sense, to the creation of a leaky pipe that cannot sustain the propagation of a pressure wave.

The pore density distribution function $n(r)$ obtained from the Smoluchowski model for a 100-kV/cm, 20-ns HISDEP and another $200-\mathrm{kV} / \mathrm{cm}, 50-\mathrm{ns}$ pulse is shown in Fig. 6. A $23.8 \mu \mathrm{m}$ diameter was assumed for the cylinder. Snapshots were taken after both pulses had terminated. Increases in pore density and a shift towards larger pore diameters for the higher-amplitude, longer-duration pulse is evident and expected. In either case, pore radii are predicted to be less than $1 \mathrm{~nm}$, underscoring the nanoscale dimensions of the electrically created pores. Since the applied electric field has an angular nonuniformity, the pore density is expected to be heterogeneous. This is shown in Fig. 7 with the density plotted as a function of angle and time for a $150-\mathrm{kV} / \mathrm{cm}$ external pulse. The electric pulse shape was taken to have a $10-n$ sise 


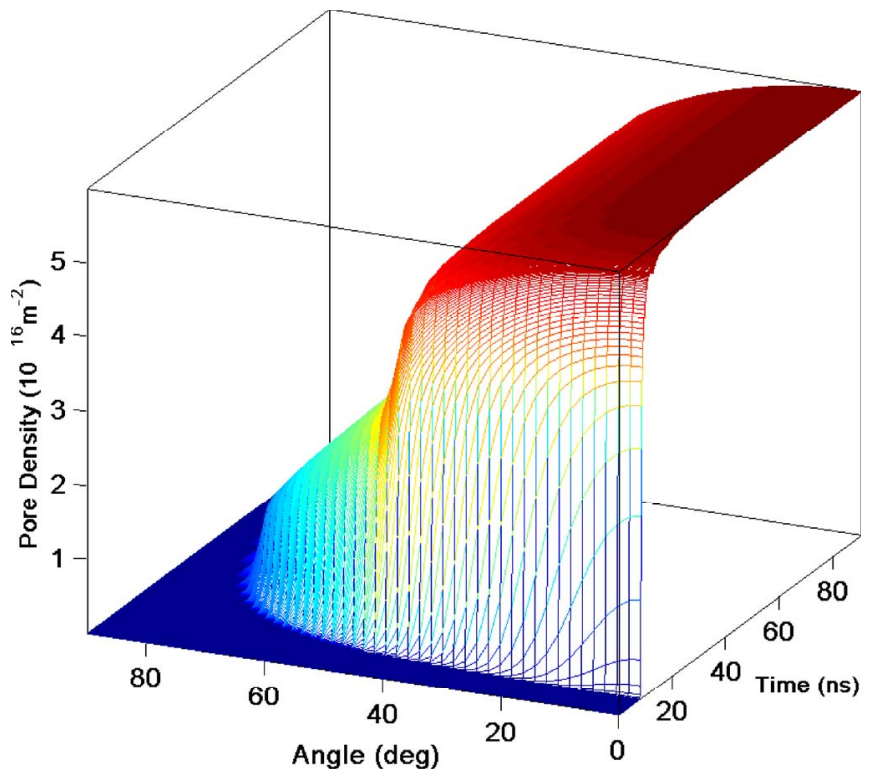

FIG. 7. (Color online) Pore density evolution for a $150-\mathrm{kV} / \mathrm{cm}$ pulse as a function of the angular distribution. The pulse had a rise time of $10 \mathrm{~ns}$, an ON time of $50 \mathrm{~ns}$, and a fall time of $10 \mathrm{~ns}$.

time, a 50-ns ON time, and a 10-ns fall time, in keeping with the local "pulsed-power" experimental systems. From consideration of symmetry, only the $0^{\circ}<\theta<90^{\circ}$ range has been shown. Clearly, the largest pore densities are created at $\theta$ $=0^{\circ}$ where the normal field is the strongest. A finite time delay for poration onset, with gradual density increases in time, is predicted for the higher angles. The characteristics of pores formed by such a nanosecond pulse lies in the creation of the larger density of smaller-sized pores. Other groups (e.g., Vasilkoski et al. [36]) term this difference as "supraporation." Physically, the pore creation rate depends nonlinearly (almost exponentially) on the transmembrane potential. Hence, the high-intensity pulses used here facilitate the creation of a high density of pores very quickly, before the pulse is turned off. This differs from the conventional, low-voltage pulses (e.g., Wilhelm et al. [75]) that favor the creation of a relatively prominent large-radius pore.

A corresponding plot of the transmembrane potential is given in Fig. 8. At the smaller angles, the voltages quickly reach values over $1.0 \mathrm{~V}$ and subsequently collapse as poration is initiated. Transmembrane potentials at the higher angles close to $\theta \sim 90^{\circ}$ show a distinct delay and remain relatively high given the absence of local poration or the generation of localized conduction currents. For small angles, the slight negative values at about $70 \mathrm{~ns}$ arise from the strong displacement currents during pulse turn-off.

Snapshots of pore densities as a function of the angular position at the end of different pulse durations for a $150-\mathrm{kV} / \mathrm{cm}$ electric field are shown in Fig. 9. This brings out the effect of varying pulse widths. The rise and fall times were kept at $10 \mathrm{~ns}$, while the on-time was varied. Due to the relatively high field intensity chosen, there is minimal variation up to about $\theta=70^{\circ}$, and almost that entire region of the membrane is well porated. A sharp drop-off in poration density is predicted beyond $\theta=80^{\circ}$. Results showing variations in pore density with electric field intensity at constant pulse

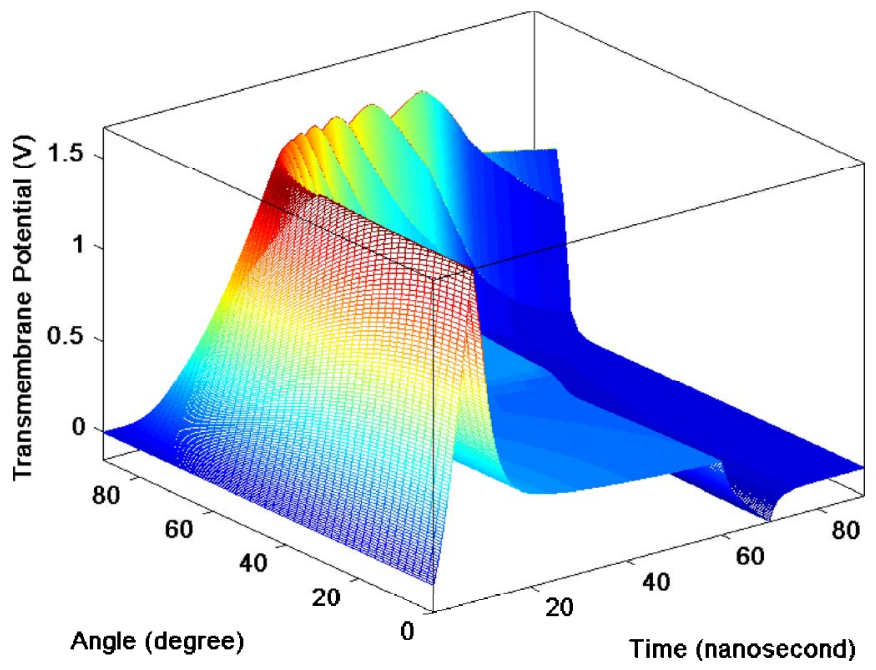

FIG. 8. (Color online) Transmembrane potential evolution for a $150-\mathrm{kV} / \mathrm{cm}, 10-\mathrm{ns}-50-\mathrm{ns}-10-\mathrm{ns}$ pulse as a function of the angle.

duration are given in Fig. 10. Again, the sharpest variations occur at the highest angles, and most of the remaining regions are predicted go be well porated, suggesting an abundant pulse-modulated membrane conductance change.

The summarizing trend from the various simulations was that the high-intensity pulses were capable of efficiently porating much of the membrane fairly uniformly. Also, the electrical stimulation created nanopores with a Gaussiantype spread in radius, rather than large openings as with the traditional low-intensity, long-duration electroporation pulses. It was also observed that using pulses as short as 20 ns would be sufficient to achieve the desired objective. Furthermore, the simulations yielded nearly identical pore densities for cylinders with variable diameters ranging from $10 \mu \mathrm{m}$ to $50 \mu \mathrm{m}$. Though all of the variable-radius data have not been shown, the results suggest a fairly robust response.

\section{Action potential evolution with membrane electroporation}

The distributed electrical model of Fig. 1 incorporates the membrane shunt conductance $G_{\text {sh }}$ and, hence, deviates from

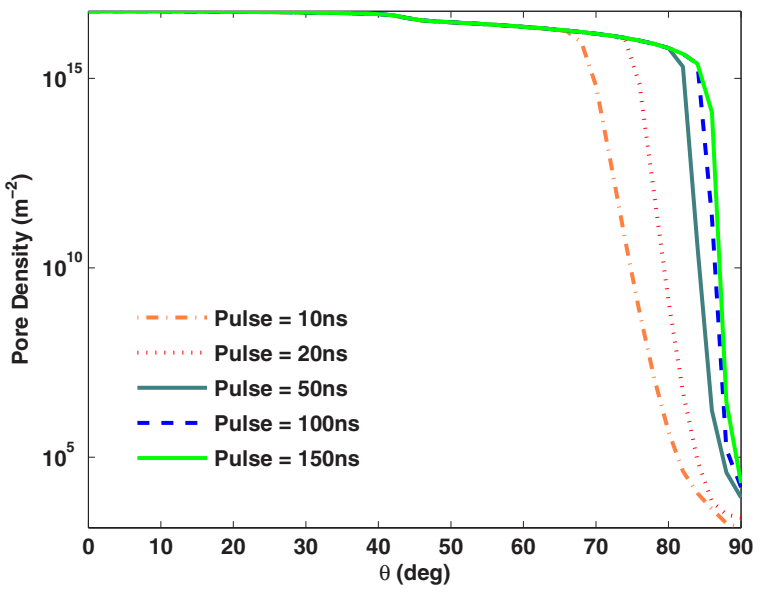

FIG. 9. (Color online) Predicted pore densities at the end of various pulse durations at $150 \mathrm{kV} / \mathrm{cm}$. 


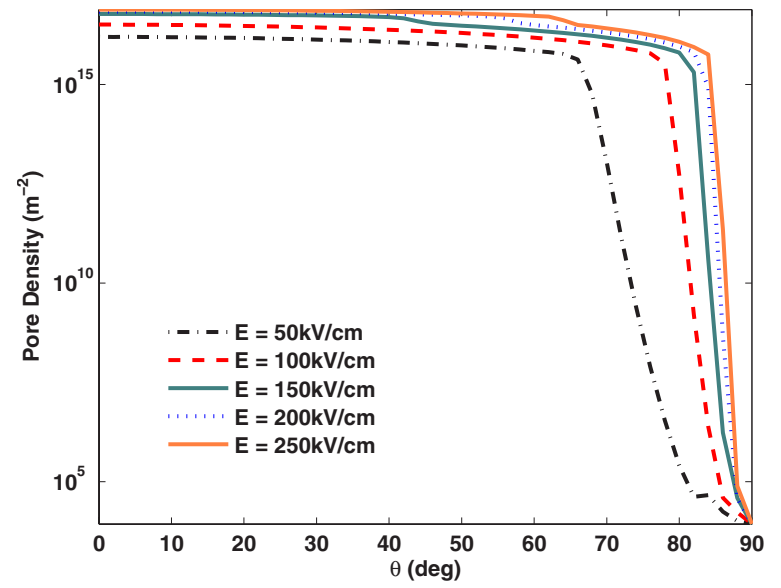

FIG. 10. (Color online) Predicted pore densities for different field intensities at constant pulse duration.

the traditional Hodgkin-Huxley [15] and HuxleyFrankenhaeuser [16] representations. It was used to simulate action potential propagation for various values of $G_{\mathrm{sh}}$ for purposes of determining the minimum value required to block neural traffic. This was done independent of the electroporation calculation of the previous section and had a twofold purpose. (a) It served to confirm and substantiate the notion of an AP block through changes in membrane conductance. (b) Second, it yielded minimum thresholds for the requisite conductance as a function of nerve diameter for $\mathrm{AP}$ blockage. The results obtained are shown in Fig. 11. The number of nodes (or nerve sections) affected by membrane poration was taken to be the $x$ variable in Fig. 11. As might be expected, the requisite conductance threshold for an AP block decreases with increasing node numbers. Physically, this implies that a lower degree of poration at a nerve segment might still be sufficient to block voltage propagation, provided a larger number of segments contributed to cumulatively satisfy the requisite current leakage. However, the outcome does not scale with the number of segments, and Fig. 11 suggests that even a relatively low number of nodes

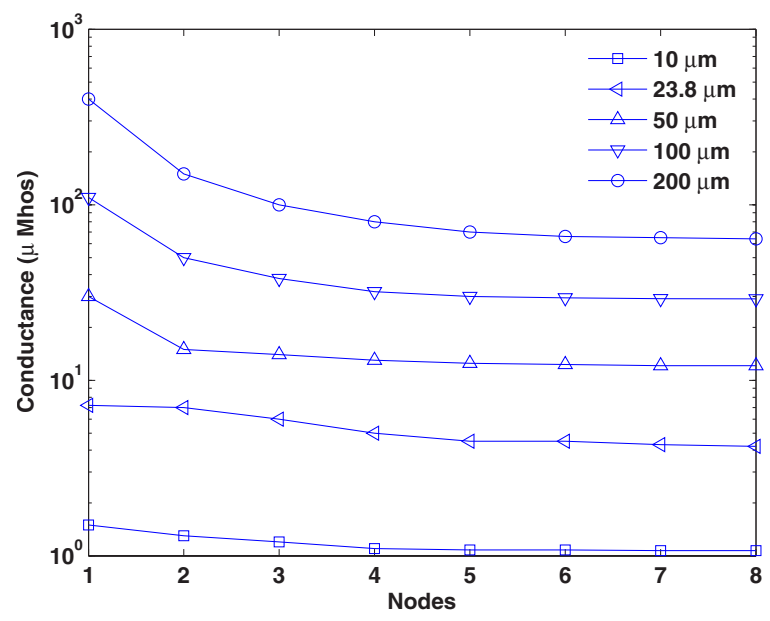

FIG. 11. (Color online) Minimum membrane shunt conductances required to block action potential propagation as a function of the number of electroporated nodes for various nerve diameters.
TABLE II. Maximum conductance $G_{\mathrm{sh}}$ computed at $100 \mathrm{mV}$ for different field amplitudes and pulse durations of the electroporating excitation.

\begin{tabular}{lccc}
\hline \hline $\begin{array}{l}\text { Maximum } \\
\text { amplitude } \\
(\mathrm{kV} / \mathrm{cm})\end{array}$ & $\begin{array}{c}\text { Pulse } \\
\text { duration (ns) }\end{array}$ & Conductance $(\mathrm{S})$ & AP Block ? \\
\hline 100.0 & 20.0 & $3.0777 \times 10^{-4}$ & Yes \\
100.0 & 50.0 & $3.8265 \times 10^{-4}$ & Yes \\
100.0 & 100.0 & $4.3744 \times 10^{-4}$ & Yes \\
150.0 & 20.0 & $5.9528 \times 10^{-4}$ & Yes \\
150.0 & 50.0 & $7.0608 \times 10^{-4}$ & Yes \\
150.0 & 100.0 & $7.8385 \times 10^{-4}$ & Yes \\
200.0 & 20.0 & $7.7152 \times 10^{-4}$ & Yes \\
200.0 & 50.0 & $9.0424 \times 10^{-4}$ & Yes \\
\hline \hline
\end{tabular}

would be sufficient for an AP block. From a practical standpoint, since such external pulses will be applied through sharp electrodes, the external electric field is likely to rapidly fall off with distance. Hence, it would be unrealistic to expect uniform poration and conductance changes along nerves. Different segments of a nerve would likely have varying $G_{\text {sh }}$ values in response to the HISDEP stimulation. The present calculation demonstrates that even with only a couple of porated segments, it would be possible to provide an AP block, provided the requisite change in $G_{\text {sh }}$ can be attained. Another aspect from Fig. 11 is the monotonic increase in shunt conductance with nerve diameter. Thus, thinner nerves are predicted to be easier for AP blocking, while thicker nerves would have more stringent HISDEP requirements.

Finally, for self-consistent calculations of AP blockage, the results of pore densities were used to obtained realistic changes in $G_{\text {sh }}$ through Eq. (16). These values depend on the membrane voltage $U(t)$ and were coupled to the $\mathrm{HH}$ distributed circuit model for self-consistent time-dependent simulations. The maximum values of $G_{\mathrm{sh}}$ obtained for a trans-membrane voltage of $104 \mathrm{mV}$ corresponding to the AP peaks of Fig. 4 for various pulse conditions are given in Table II. The electric field intensity ranged from 100 to $200 \mathrm{kV} / \mathrm{cm}$, while the pulse duration was from 20 to $100 \mathrm{~ns}$. In all cases, AP blockage was achieved. A representative result is shown in Fig. 12. Nerve voltage at various nodes versus time is shown for an AP launched from node 2. A 20-ns, $100-\mathrm{kV} / \mathrm{cm}$ HISDEP was taken to be applied at node 25 . Use of the corresponding $G_{\mathrm{sh}}$ obtained yielded a complete blockage of the action potential.

On a related note, it is perhaps germane to briefly discuss the effective duration of the AP conduction blockage. As long as the pores do not reseal, or even shrink considerably in size and population, the neural traffic can be blocked at a nerve. Resealing typically would follow an exponential decay $[36,43]$, with resealing times on the order of seconds or longer are expected. Hence, HISDEP pulsing with a $1-2-\mathrm{Hz}$ repetition rate would be sufficient to achieve prolonged blockage without any deleterious effects of heating-related tissue damage or self-activated AP launches. Also, in this 


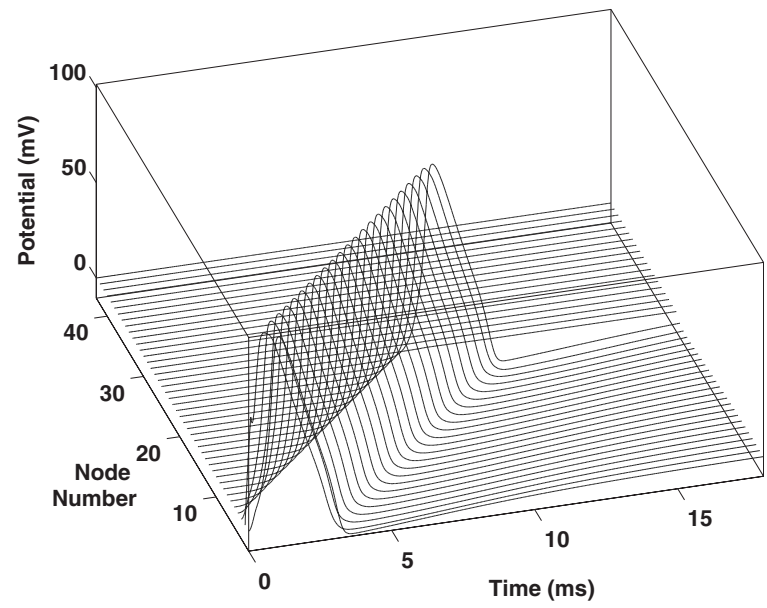

FIG. 12. Simulations showing node voltage versus time for an AP launched from node 2. A 20-ns, 100-kV/cm HISDEP was taken to be applied at node 25. An action potential block is seen.

contribution, we have focused primarily on extremely high electric fields. It is conceivable that at reduced strengths, other effects such as the activation of voltage-gated channels would begin to play a role and influence the neural propagation characteristics. Studies of such effects will be reported elsewhere.

\section{CONCLUSIONS}

It is shown that high-intensity, short-duration electrical pulses could provide for a quick-acting, localized, and reversible cessation of biological electrical signaling pathways. This would have obvious applications in neurophysiology, clinical research, neuromuscular stimulation therapies, and neuromuscular disruption leading to possible nonlethal bioweapons. The concept of arresting action potential propaga- tion on command through external electrical stimulation is based on creating a large density of pores on the membrane of axons. The advantages of such an approach include reversibility, suppression of possible self-launched action potentials, and negligible heating or tissue damage.

A self-consistent theoretical analysis has been performed. The continuum approach based on the Smoluchowski equation was used to assess membrane electroporation by a highintensity external pulse. The angular and temporal aspects were considered on an equal footing, and details of pore formation and growth in $r$ space were also included. Changes in the membrane potential due to conduction current flows arising from localized electroporation were also considered.

Our results indicate that a sufficiently high density of pores can be generated by a high-intensity, nanosecond electric pulse. The resulting change in membrane conductance then presents an effective "electrical short" to an incident voltage wave traveling across the nerve. The net effect is that the local membrane potential at the affected node is unable to rise significantly. This prevents current injection and activation of sodium channels downstream, thereby blocking AP propagation. It has also been shown that poration at a single neural segment would be sufficient to produce an observable effect. In reality, more than one segment would be affected. Also, the influence of external electric fields would be better assessed by taking account of nonuniformities and spatial distributions of the potential between nerves and the surface electrode regions.

\section{ACKNOWLEDGMENTS}

This work was sponsored in part by an AFOSR-MURI grant No. (F49620-02-1-0320) on Subcellular Responses to Narrowband and Wideband Radio Frequency Radiation managed by Dr. Robert J. Barker. The authors would like to thank J. Weaver (MIT), S. Beebe (EVMS), J. Kolb (ODU), and R. Nuccitelli (ODU) for useful discussions.
[1] N. Bhadra and K. Kilgore, IEEE Trans. Neural Syst. Rehabil. Eng. 12, 313 (2004); K. Kilgore and N. Bhadra, Med. Biol. Eng. Comput. 12, 394 (2004).

[2] J. C. Petruska, C. H. Hubscher, and R. D. Johnson, Exp. Brain Res. 121, 379 (1998).

[3] C. Tai, W. C. de Groat, and J. R. Roppolo, IEEE Trans. Biomed. Eng. 52, 1323 (2005).

[4] M. Manfredi, Arch. Ital. Biol. 108, 52 (1970).

[5] J. G. Whitwam and C. Kidd, Br. J. Anaesth. 47, 1123 (1975).

[6] M. Cattell and R. W. Gerard, J. Physiol. (London) 83, 407 (1935).

[7] A. Rosenblueth and J. Reboul, Am. J. Physiol. 125, 251 (1939).

[8] S. W. Kuffler and R. W. Gerard, J. Neurophysiol. 10, 383 (1947).

[9] A. S. Paintal, J. Physiol. (London) 180, 1 (1965).

[10] M. Ishigooka, T. Hashimoto, I. Sassagawa, K. Izumiya, and T. Nakada, Eur. Urol. 25, 334 (1994).
[11] S. Schumacher, S. Bross, J. R. Scheepe, C. Seif, K. P. Junemann, and P. Alken, J. Urol. (Baltimore) 161, 950 (1999).

[12] S. J. Tsai, H. L. Lew, E. Date, and L. I. Bih, Arch. Phys. Med. Rehabil. 83, 714 (2002).

[13] R. Baratta, M. Ichie, S. K. Hwang, and M. Solomonow, IEEE Trans. Biomed. Eng. 36, 836 (1989).

[14] Z. P. Fang and J. T. Mortimer, IEEE Trans. Biomed. Eng. 38, 175 (1991).

[15] A. L Hodgkin and A. F. Huxley, J. Physiol. (London) 117, 500 (1952).

[16] B. Frankenhaeuser and A. F. Huxley, J. Physiol. (London) 171, 302 (1964).

[17] J. P. Reilly, V. T. Freeman, and W. D. Larkin, IEEE Trans. Biomed. Eng. 32, 1001 (1985).

[18] F. Rattay, J. Theor. Biol. 123, 45 (1986).

[19] P. H. Veltink, J. A. V. Alste, and H. B. K. Boom, IEEE Trans. Biomed. Eng. 35, 69 (1988).

[20] K. W. Altman and R. Plonsey, IEEE Trans. Biomed. Eng. 37, 
688 (1990)

[21] For example, W. F. Agnew and D. B. McCreery, Neural Prosthesis: Fundamental Studies (Prentice Hall, Englewood Cliffs, 1990).

[22] N. Acconero, G. Bini, G. L. Lenzi, and M. Manfredi, J. Physiol. (London) 273, 539 (1977).

[23] A. G. Brown and W. C. Hamann, J. Physiol. (London) 222, 66 (1972).

[24] K. H. Schoenbach, F. E. Peterkin, R. W. Alden, and S. J. Beebe, IEEE Trans. Plasma Sci. 25, 284 (1997).

[25] K. H. Schoenbach, S. J. Beebe, and E. S. Buescher, Bioelectromagnetics (N.Y.) 22, 440 (2001).

[26] R. P. Joshi, Q. Hu, R. Aly, K. H. Schoenbach, and H. P. Hjalmarson, Phys. Rev. E 64, 011913 (2001).

[27] K. H. Schoenbach, R. P. Joshi, J. Kolb, N. Chen, M. Stacey, P. Blackmore, E. S. Buescher, and S. J. Beebe, Proc. IEEE 92, 1122 (2004).

[28] P. T. Vernier, Y. Sun, L. Marcu, S. Salemi, C. M. Craft, and M. A. Gundersen, Biochem. Biophys. Res. Commun. 310, 286 (2003).

[29] S. J. Beebe, P. F. Blackmore, J. White, R. P. Joshi, and K. H. Schoenbach, Physiol. Meas 25, 1077 (2004).

[30] A. J. H. Sale and W. A. Hamilton, Biochim. Biophys. Acta 148, 781 (1967); 163, 37 (1968).

[31] K. H. Schoenbach, R. P. Joshi, R. H. Stark, F. C. Dobbs, and S. J. Beebe, IEEE Trans. Dielectr. Electr. Insul. 7, 637 (2000).

[32] R. Nuccitelli, U. Pliquett, X. Chen, W. Ford, R. J. Swanson, S. J. Beebe, J. F. Kolb, and K. H. Schoenbach, Biochem. Biophys. Res. Commun. 343, 351 (2006).

[33] M. Stacey, J. Stickley, P. Fox, V. Statler, K. H. Schoenbach, S. J. Beebe, and S. Buescher, Mutat Res. 542, 65 (2003).

[34] R. Nuccitelli, Radiat. Prot. Dosim. 106, 375 (2003).

[35] M. Zhao, B. Song, J. Pu, T. Wada, B. Reid, G. Tai, F. Wang, A. Guo, P. Walczysko, Y. Gu, T. Sasaki, A. Suzuki, J. V. Forrester, H. R. Bourne, P. N. Devreotes, C. D. McCaig, and J. M. Penninger, Nature (London) 442, 457 (2006).

[36] Z. Vasilkoski, A. T. Esser, T. R. Gowrishankar, and J. C. Weaver, Phys. Rev. E 74, 021904 (2006).

[37] J. C. Neu and W. Krassowska, Phys. Rev. E 67, 021915 (2003).

[38] R. P. Joshi, Q. Hu, and K. H. Schoenbach, IEEE Trans. Dielectr. Electr. Insul. 10, 778 (2003).

[39] R. P. Joshi, Q. Hu, K. H. Schoenbach, and H. P. Hjalmarson, Phys. Rev. E 65, 041920 (2002).

[40] Q. Hu, R. P. Joshi, and K. H. Schoenbach, Phys. Rev. E 72, 031902 (2005).

[41] Q. Hu, S. Viswanadham, R. P. Joshi, K. H. Schoenbach, S. J. Beebe, and P. F. Blackmore, Phys. Rev. E 71, 031914 (2005).

[42] D. Tieleman, H. Leontiadou, A. E. Mark, and S. J. Marrink, J. Am. Chem. Soc. 125, 6382 (2003).

[43] S. Koronkiewicz, S. Kalinowski, and K. Bryl, Biochim. Biophys. Acta 1561, 223 (2002).

[44] M. Lambreva, B. Glück, M. Radeva, and H. Berg, Bioelectro- chemistry 62, 95 (2004).

[45] G. Saulis, M. S. Venslauskas, and J Naktinis, Bioelectrochem. Bioenerg. 26, 1 (1991).

[46] A. Zhou, M. Liu, Zhou, C. Baciu, B. Glück, and H. Berg, J. Electroanal. Chem. 486, 220 (2000).

[47] K. A. Riske and R. Dimova, Biophys. J. 88, 1143 (2005).

[48] K. Ohuchi, Y. Fukui, I. Sakuma, N. Shibata, H. Honjo, and I. Kodama, IEEE Trans. Biomed. Eng. 49, 18 (2002).

[49] D. R. McNeal, IEEE Trans. Biomed. Eng. 23, 329 (1976).

[50] J. W. Cooley and F. A. Dodge, Jr., Biophys. J. 6, 583 (1966).

[51] F. Rattay, IEEE Trans. Biomed. Eng. 33, 974 (1986).

[52] J. T. Rubinstein, Biophys. J. 68, 779 (1995).

[53] J. C. Weaver and R. A. Mintzer, Phys. Lett. 86A, 57 (1981).

[54] V. F. Pastushenko and Yu. A. Chhizmadzhev, Biofizika 28, 1036 (1983); 27, 475 (1982).

[55] V. F. Pastushenko, Yu. A. Chhizmadzhev, and V. B. Arakelyan, Bioelectrochem. Bioenerg. 6, 53 (1979).

[56] A. Barnett and J. C. Weaver, Bioelectrochem. Bioenerg. 25, 163 (1991).

[57] S. A. Freeman, M. A. Wang, and J. C. Weaver, Biophys. J. 67, 42 (1994).

[58] J. C. Neu and W. Krassowska, Phys. Rev. E 59, 3471 (1999).

[59] M. Winterhalter and W. Helfrich, Phys. Rev. A 36, 5874 (1987).

[60] T. Y. Tsong, Biophys. J. 60, 297 (1991).

[61] E. Neumann, E. Sowers, and C. A. Jordan, Electroporation and Electrofusion in Cell Biology (Plenum Press, New York, 1989).

[62] E. Neumann and K. Rosenheck, J. Membr. Biol. 10, 279 (1972).

[63] J. C. Weaver, Ann N.Y. Acad. Sci 720, 141 (1994).

[64] J. Teissie and M. P. Rols, Biophys. J. 58, 1089 (1990).

[65] R. W. Glaser, S. L. Leikin, L. V. Chernomordik, V. F. Pastushenko, and A. I. Sokirko, Biochim. Biophys. Acta 940, 275 (1988).

[66] I. G. Abidor, V. B. Arakelyan, L. V. Chernomordik, Y. A. Chizmadzhev, V. F. Pastushenko, and M. R. Tarasevich, Bioelectrochem. Bioenerg. 6, 37 (1979).

[67] J. C. Weaver and Yu. A. Chizmadzhev, Bioelectrochem. Bioenerg. 41, 135 (1996).

[68] A. Barnett, Biochim. Biophys. Acta 1025, 10 (1990).

[69] L. V. Chernomordik, S. I. Sukharev, S. V. Popov, I. G. Abidor, V. F. Pastushenko, A. V. Sokirko, and Y. A. Chizmadzhev, Biochim. Biophys. Acta 902, 360 (1987).

[70] J. E. Hall, J. Gen. Physiol. 66, 531 (1975).

[71] B. Hille, Ionic Channels of Excitable Membranes (Sinauer, Sunderland, 2001); B. Hille, J. Gen. Physiol. 51, 199 (1968).

[72] C. B. Muratov, Biophys. J. 79, 2893 (2000).

[73] C. C. McIntyre, A. G. Richardson, and W. M. Grill, J. Neurophysiol. 87, 995 (2002).

[74] R. N. Miller and J. Rinzel, Biophys. J. 34, 227 (1981).

[75] C. Wilhelm, M. Winterhalter, U. Zimmermann, and R. Benz, Biophys. J. 64, 121 (1993). 